\title{
Design of Spread Spectrum Multicode CDMA Transport Architecture for Multimedia Services
}

\author{
Po-Rong Chang, Member, IEEE, and Chin-Feng Lin
}

\begin{abstract}
In this paper, we investigate a new application of the well-known spread spectrum code division multiple access (SS-CDMA) techniques to multimedia services related to the development of the next-generation wireless mobile networks interconnecting with a wireline ATM-based broadband network. Such services allow users to share novel multimedia applications without any geographical restrictions. However, since the mobile radio channel has a fixed limited bandwidth, the traditional SS-CDMA system may not be sufficient to accommodate the variable bit rate (VBR) multimedia services requested by multiple mobile users simultaneously. Moreover, the traffic load at the base station can change dynamically due to the time-varying throughput requirement of these requested multimedia services. To tackle this difficulty, a multicode CDMA (MC-CDMA) technique is proposed to provide multirate multimedia services by varying the number of spreading codes assigned to each user in order to meet its throughput requirement. In MC-CDMA, a spreading code can be used to transmit information at a basic bit rate. Users (video or data) who need higher transmission rates can use multiple codes in parallel. Meanwhile, the maximum available number of codes in the MC-CDMA system is still limited. Hence, a cost-effective dynamic code allocation scheme has then been proposed to dynamically assign appropriate number of codes to each user for achieving the maximum resource utilization for multiuser multimedia services via the mobile radio channel. Finally, a number of real multimedia titles generated from the well-known MacroMind Director are conducted to evaluate the performance of the proposed wireless multimedia CDMA system.
\end{abstract}

Index Terms-Multicode code division multiple access (CDMA), multimedia, object-composition Petri-net (OCPN).

\section{INTRODUCTION}

$\mathbf{T}$ I HE THIRD-GENERATION mobile communication system currently being developed is intended to integrate all the different services of second-generation systems and cover a much wider range of broadband services (audio, data, video, multimedia) consistent and compatible with the technology developments taking place within the broadband ISDN wireline networks [1], [2]. The concept of wireless mobile communication networks suggests itself as a replacement for wired multimedia communication networks in order to avoid expensive installation and relocation and to provide portability and mobility to various pieces of equipment. Furthermore, the use of radio for mobile multimedia communication in

Manuscript received October 2, 1998; revised April 17, 1999. This work was supported in part by the National Science Council, Taiwan, R.O.C under Contract NSC-87-2218-E-009-046.

The authors are with the Department of Communication Engineering, National Chiao-Tung University, Hsin-Chu, Taiwan, R.O.C. (e-mail: prchang@cc.nctu.edu.tw).

Publisher Item Identifier S 0733-8716(00)00191-8. cars, remote hospitals, military services, homes, and office automation systems is an attractive proposition. It would free the users from cords or optical fibers tying them to particular locations within the building, thus offering true mobility which convenient and sometimes even necessary. The development of multimedia terminals will support the ever-growing demand for mixed data, audio, and video applications and will connect the portable pen pad and lap-top devices to backbone information resources and computational facilities. The possibility of multimedia services will allow services such as dial-up video conference, video-on-demand (VOD) services, and portable PC-based applications incorporating video/audio/data transfer to any location. Moreover, a number of different mobile users can simultaneously request multimedia data from one or more multimedia servers on the network. Each multimedia server is capable of catering to multiple data requests from multiple users, simultaneously. Presentation of preorchestrated multimedia information requires synchronous playback of time-dependent multimedia data according to some prespecified temporal relations. At the time of creation of multimedia information, a user needs a model to specify temporal constraints among various data objects which must be observed at the time of playback. Usually, the temporal relationships of multimedia information may be characterized by a timeline diagram which is the commonly used tool in commercial multimedia authoring products. Fig. 1 depicts an example of a timeline diagram and its associated multimedia title generated by the most commonly used product called MacroMind Director. Although the timeline diagram is a useful description tool, it has a lot of redundancies in characterizing the temporal relationships and is not suitable for further analysis and system evaluation, however. To tackle this difficulty and to obtain a more compact multimedia representation, a well-known model called object-composition Petri-net (OCPN) [3], [4] is able to describe the temporal relationships of the various components of a multimedia document and represents them in the form of a graph. Since preorchestrated multimedia information has highly time-varying bandwidth, the fixed bandlimited constant bit rate (CBR) wireless channel may not be appropriate for the variable bit rate (VBR) multimedia services. Therefore, it is desirable to design a dynamic mechanism to manage and allocate bandwidth according to the changing levels of concurrencies of multimedia data streams. Woo et al.[5] have introduced a dynamic RF channel capacity allocation to deal with the OCPN-based multimedia data stream. In this paper, an alternative method has been proposed to provide a cost-effective resource allocation scheme for the OCPN-based multimedia services by employing the well-known antimultipath spread 


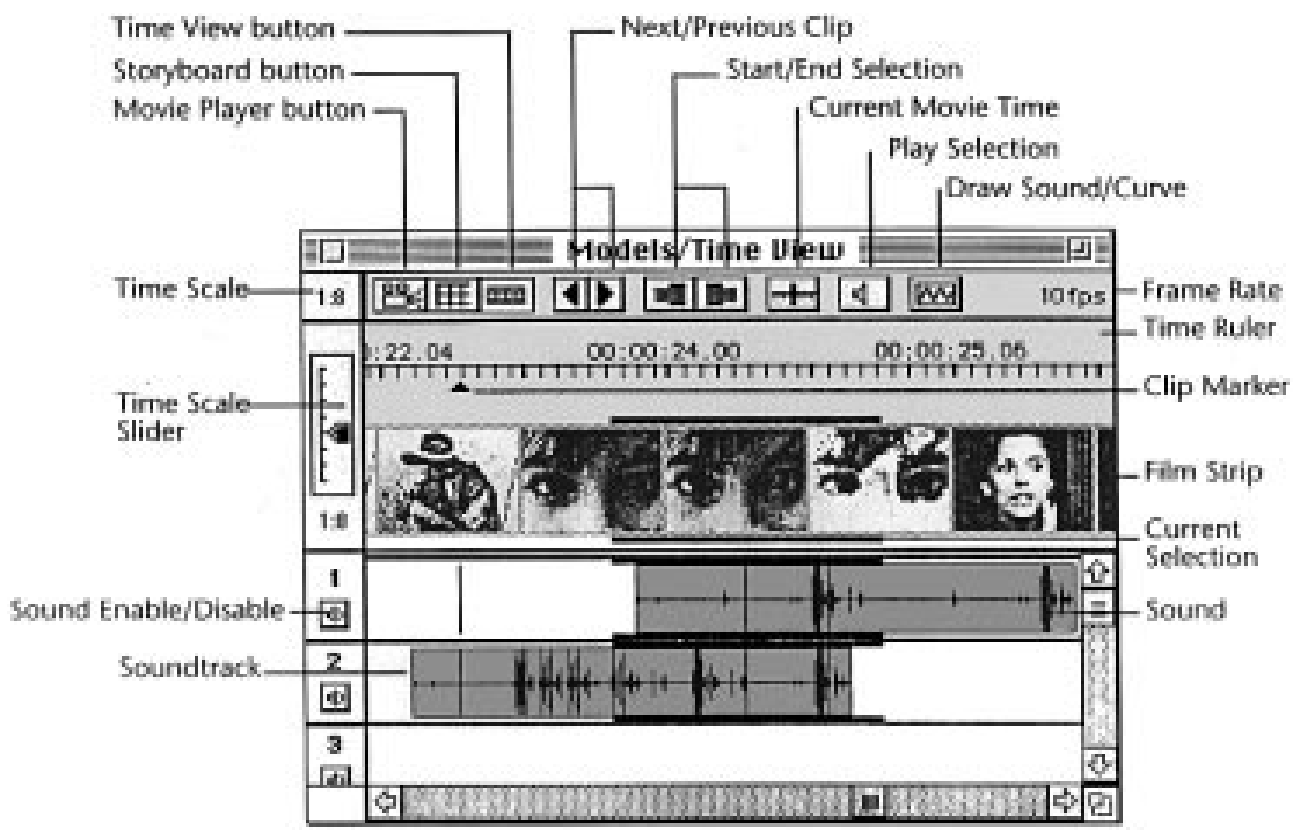

Fig. 1. Timeline diagram of a multimedia title generated from MacroMind Director.

spectrum code division multiple access (SS-CDMA) techniques.

In terms of the multiplexing/multiple access techniques, CDMA has gained significant attention in recent years as a competing technology for wireless mobile networks [6]. Unfortunately, a major weakness of the conventional CDMA is that for a given system bandwidth, spectrum-spreading limits the peak user data rate to a relatively low value. For example, even with $50 \mathrm{MHz}$ bandwidth and a spreading gain of 512, user bit rate is limited to just $100 \mathrm{~kb} / \mathrm{s}$. Meanwhile, multimedia services should be supported with a high transmission bit rate within a wider allowable bandwidth. This problem could be ameliorated via multiple code CDMA transmission per user [7]-[9], [15]. In other words, higher data rates are achieved by allocating more than one code to a single user in order to create more than one virtual channel for the user. The code number assigned to each user is proportional to the dynamic throughput requirement of multimedia services requested by the user. However, since the maximum available code number is limited, total code number requirements may exceed the available number. Therefore, a dynamic code allocation mechanism has been proposed to assign an appropriate code number to each user in order to achieve the maximum resource utilization for the OCPN-based multiuser multimedia services. Furthermore, in order to avoid the self-interference that a user employing multiple codes may incur, the multiple codes to/from one user should be made orthogonal. This particular spread-coding scheme is called the concatenated orthogonal/PN spreading code [7]-[9], which is capable of subdividing a high rate stream belonging to a user into several parallel lower basic bit rate streams.

\section{OBject COMPosition Petri-Net (OCPN) Models FOR MULTIMEDIA INFORMATION}

A form of timed Petri nets called the OCPN has been used in [3] and [4] to specify synchronization and the relationship between multimedia objects. The OCPN has been demonstrated to capture all possible temporal relationships between objects required for multimedia presentation. Formally, the OCPN is defined as a bipartite directed graph $N_{\text {OCPN }}$, specified by the tuple $\{\boldsymbol{T}, \boldsymbol{P}, \boldsymbol{A}, \boldsymbol{D}, \boldsymbol{R}, \boldsymbol{M}\}$ where:

$$
\begin{aligned}
& \boldsymbol{T}=\left\{t_{1}, t_{2}, \ldots, t_{m_{1}}\right\} \\
& \text { set of transitions (bars); } \\
& \boldsymbol{P}=\left\{p_{1}, p_{2}, \ldots, p_{m_{2}}\right\} \\
& \text { set of places (circles); } \\
& \boldsymbol{A}=\{\boldsymbol{T} \times \boldsymbol{P}\} \cup\{\boldsymbol{P} \times \boldsymbol{T}\} \rightarrow I^{+}, I^{+}=\{1,2, \ldots\} \\
& \quad \text { set of directed arcs; }
\end{aligned}
$$$$
D: P \longrightarrow R
$$

mapping from the set of places to the real numbers (time durations);

$\boldsymbol{R}: \boldsymbol{P} \rightarrow\left\{r_{1}, r_{2}, \ldots, r_{m_{3}}\right\}$

mapping from the set of place to a set of object types, corresponding to video, audio, or textural objects.

$$
M: \boldsymbol{P} \rightarrow I^{0}, I^{0}=\{0,1,2, \ldots\}
$$

mapping from the set of place to the integer, which represents the number of tokens in a specified place.

Associated with the definition of the Petri-net is a number of firing rules governing the semantics of the model. A transition $t_{i}$ fires immediately when each of its input places contains an unlocked token. Upon firing, the transition $t_{i}$ removes a token from each of its input places and adds a token to each of its output places. After receiving a token, a place $p_{i}$ remains in the active state for the time interval specified by the duration $\tau_{i}$. During the interval, the token is locked. When the place becomes inactive, or upon expiration of the duration $\tau_{i}$, the token becomes unlocked. For multimedia information modeling, a place $p_{i}$ in an OCPN represents the playout process of multimedia object $O_{i}$. Attributes associated with the object include its type, size, throughput requirements, and the duration of its presentation. Moreover, a transition in an OCPN represents a synchronization point as it marks the playout start time of new concurrent objects. 


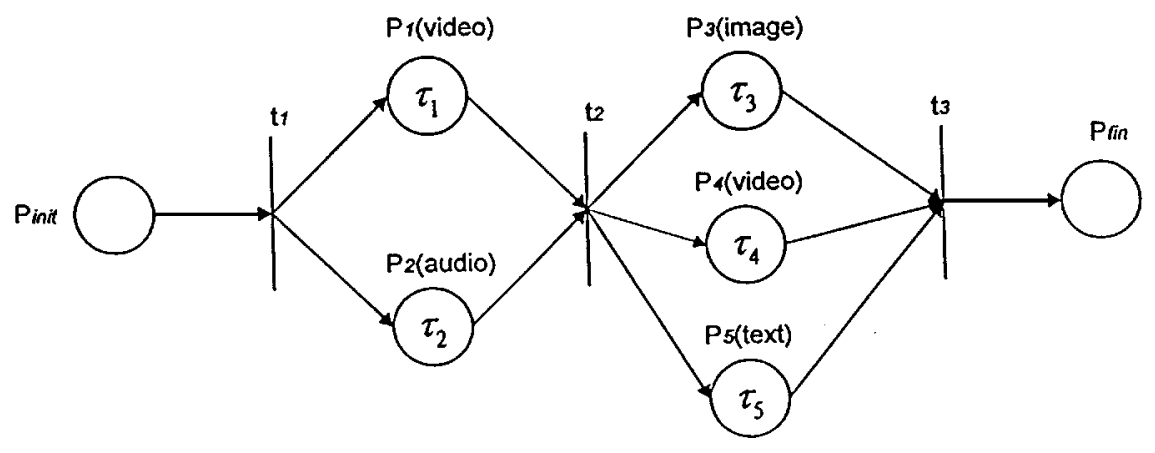

Fig. 2. Example of OCPN representation for a multimedia title.

Fig. 2 illustrates an example of OCPN. In this figure, $p_{\text {init }}$ represents the initial place in the OCPN which indicates the start of the multimedia presentation, and $p_{\mathrm{fin}}$ denotes the final place which indicates the completion of presentation. Places $p_{1}$ and $p_{4}$ correspond to the playout processes of some video data; places $p_{2}$ and $p_{5}$ correspond to the audio and text data, respectively; and $p_{4}$ plays out an image data when it has a token.

Since intermedia synchronization is ultimately desired, to synchronize multiple data streams of an OCPN, it requires concurrent streams to be played out at identical synchronization times $\pi_{i}$. Such a playout time instant is called the playout deadline. Little and Ghafoor [3] have proposed a serialize-net algorithm to determine those playout deadlines $\left\{\pi_{i}\right\}$ based on the transitions $\left\{t_{i}\right\}$ and the playout durations $\left\{\tau_{i}\right\}$. Hence, each object in the OCPN is associated with the playout deadline $\pi$ and the playout duration $\tau$.

Moreover, the OCPN, being only a specification model for presentation of multimedia information, does not contain the communication and synchronization requirements over a network. Various multimedia data types have different performance requirements for network transmission and playback at the destination. However, the rates of communication and presentation of an isochronous object, such as video or audio, need to be equal in order to provide continuity in playback. In order to achieve this purpose, isochronous objects can be divided into smaller units of information to be used for maintaining synchronization. The smallest unit is referred as a synchronization interval unit (SIU) [4]. As an example, the synchronization interval for a video object can be 1/30th of a second, which corresponds to the playback duration of a single video frame. Hence, a video frame represents an SIU. For audio data, its SIU can be audio sample. As a result, a complete multimedia object is transmitted as a stream of SIU's.

\section{TRANSMission OF OCPN-BASED MUltimedia INFORMATION STREAMS VIA MULTICODE CDMA MobILE COMMUNICATION NETWORKS}

In the future, the mobile communication network will allow high mobility users to access multimedia information stored at various network sites. These multimedia servers are connected over a B-ISDN wireline network. A base station serves an interface between the wireline and the mobile networks. Fig. 3 shows this concept. A server retrieves the requested multimedia data from its databases and communicates it on multiple virtual channels (MVCS) to the base station and has the connection to the requesting mobile user via mobile multipath fading channels. Each connection between the multimedia server and the base station consists of multiple virtual channels, each of which is used to transmit an object of multimedia type. The buffers at the base station provide temporary storage for some fraction of the multimedia data objects, in order to compensate for the rate difference between the wireline and wireless network. These buffers also smooth the jitter delay that occurs inside the wireline network. In the multiple access system for multimedia transmission of interest, the system is assumed to multiplex a set of multimedia objects by applying CDMA techniques to the mobile networks. Each CDMA spreading code is able to create a virtual channel for multimedia transmission via the mobile channel. Since the mobile radio channel has a fixed limited channel bandwidth, the traditional CDMA system may not be sufficient to accommodate all the VBR multimedia objects. Moreover, the traffic load at the base station can change dynamically due to various factors, such as the throughput requirement of each object, the changing level of concurrency of objects in OCPN's, and the number of users concurrently served by the base station. To overcome this difficulty, a multicode CDMA technique is applied to the multimedia transmission in order to increase its transmission bit rate via the bandlimited mobile channels. In the multicode CDMA system, when a user needs $N$ times the basic source rate, it converts its associated concurrent multimedia objects using a multiplexer into $N$ basic rate streams, encodes each with a different code, modulates them with a different Walsh modulator, and superimposes them before upconverting for mobile transmission. In other words, each code in the multicode CDMA carries a basic rate $r_{B}$.

$N$ codes in parallel will provide a single user $N$ times the basic rate capability. Note that each user admitted into the system has a primary PN code assigned to it. These PN codes are not orthogonal between users. To avoid the self-interference that a user employing multiple codes may incur, the multiple codes to/from one user should be made orthogonal. If $c_{k}$ is the primary PN code of user $k$ with a basic transmission bit rate $r_{B}$, and the $i$ th object $O_{i}$ of the user requires a throughput of $\left|O_{i}\right|, N_{k}$ new concatenated codes, $a_{k m}$ 's, can be derived from $c_{k}$ by $a_{k m}=c_{k} \times d_{m}, 1 \leq m \leq N_{k}$, where $d_{l} \perp d_{m}$, $l \neq m$, and $N_{k}=\sum_{i=1}^{l_{c}}\left\lceil\left|O_{i}\right| / r_{B}\right\rceil,\lceil x\rceil$ denotes the smallest integer greater than $x$, and $l_{c}$ is the level of concurrent objects. It should be mentioned that the total number $N_{k}$ of orthogonal 


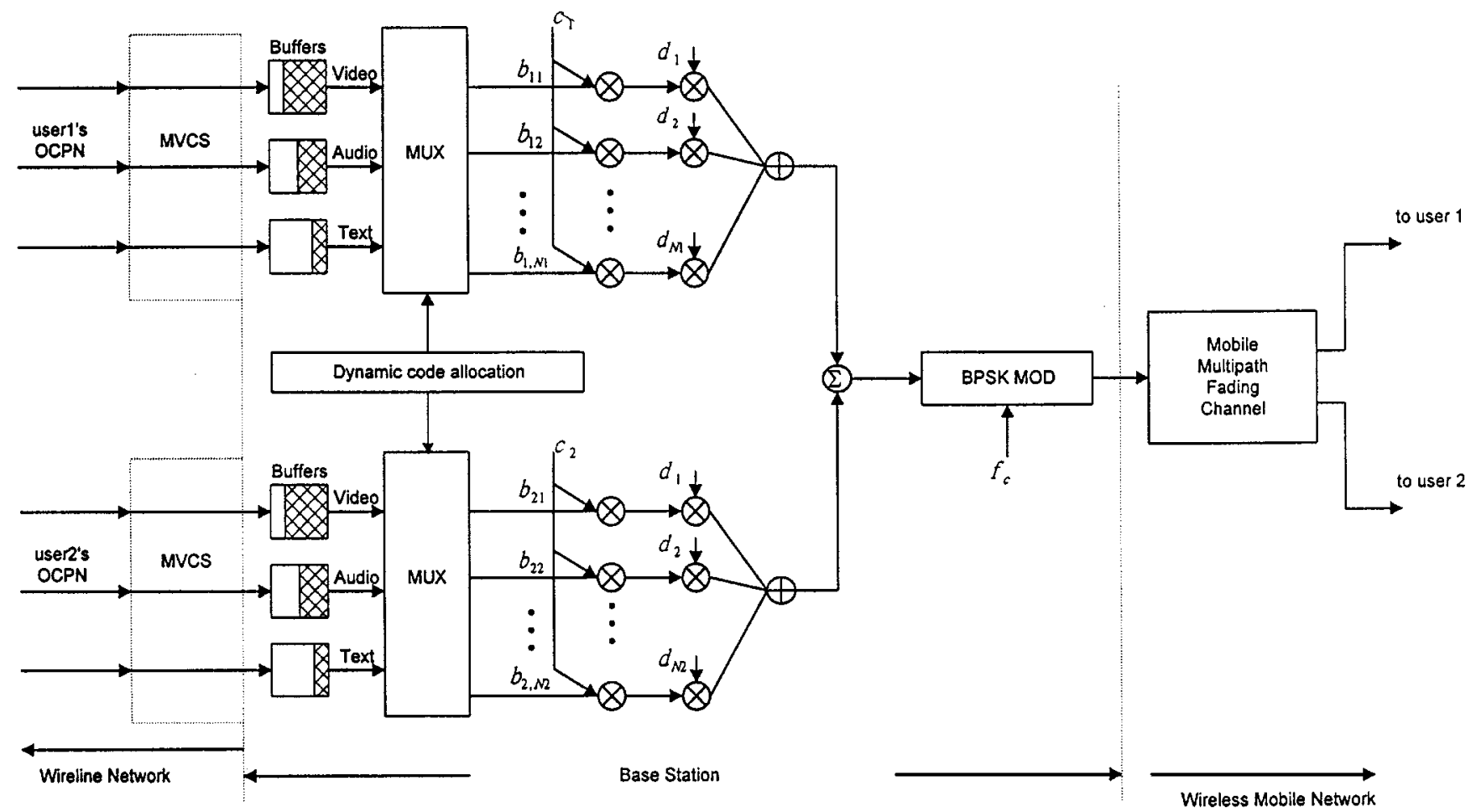

Fig. 3. Overall schematics of multimedia transmissions (downlink) via multicode CDMA mobile multipath fading channels interconnecting with wireline networks for two users, MVCS represents the multiple virtual channels, and $c_{k}$ and $d_{m}$ denote the PN and orthogonal Walsh-Hadamard (WH) codes, respectively.

codes assigned to user $k$ depends on both the throughput requirement of each object and the level of concurrent objects in a specific time interval. Due to the orthogonality requirement, the maximum number of orthogonal codes per user is the ratio $N_{c}$ of the channel chip rate and the Walsh modulator output rate. $N_{c}$ is termed as the spreading-sequence length. Hence, $N_{k} \leq N_{c}$. The previous coding strategy is called the subcode concatenated scheme that orthogonal sequences are concatenated with a PN sequence to increase the randomness of the orthogonal sequence. The binary orthogonal sequences used in this paper were the well-known Walsh-Hadamard (WH) codes which have zero cross correlation at zero time delay. They are used when synchronization of transmission can be maintained. Unfortunately, the multipath fading in a cellular radio environment introduces nonzero time delays that destroy the orthogonality between WH codes. Fong et al. [7] showed that a sufficiently long PN sequence is concatenated with WH codes to randomize and eliminate their unsatisfactory and inhomogeneous behavior at nonzero time delays. The long $\mathrm{PN}$ sequence may be chosen as either an $m$-sequence or Gold sequence.

\section{A. Multicode CDMA Transmitter Model with Concatenated Orthogonal/PN Spreading Code Scheme}

For $K$ multimedia OCPN's transmitted over a mobile channel, each of them is divided into $N_{k}(t)$ parallel data streams (virtual channels) for user $k$ at time $t$, where a specific WH code is assigned to each virtual channel. The value of $N_{k}(t)$ is dynamically proportional to both the throughput requirement of user $k$ and the level of concurrent objects belonging to user $k$ at time $t$. For simplicity, $N_{k}(t)$ is assumed to be a constant
$N_{k, j_{k}}$ in the $j_{k}$ th time interval $I_{k j_{k}}=\left[T_{k j_{k}}, T_{k, j_{k}+1}\right)$, i.e., $N_{k}(t)=N_{k j_{k}}$ for $t \in I_{k j_{k}}$. Hence, the total number of WH codes assigned to all the $K$ users in the new time interval $I_{j}$ is $M=\sum_{k=1}^{K} N_{k j_{k}}$ where $I_{j}$ denotes the $j$ th intersection interval of the time intervals for all the $K$ OCPN's, i.e., $I_{j}=\cap_{k=1}^{K} I_{k j j_{k}}$. Note that WH codes assigned to different users may be identical. It should be mentioned that the earlier arguments are valid when each user has a continuous multimedia presentation. However, this assumption may not be true since some users may not have multimedia objects during a specific time interval, i.e., $I_{k j_{k}}$. To tackle this difficulty, the value of $N_{k j_{k}}$ for this particular interval is set to zero. Therefore, the earlier argument becomes valid again. For simplicity, we assume that each of the $K$ OCPN's has its corresponding multimedia objects during $I_{j}$. The transmitting binary phase shift keying (BPSK) signal $s_{k m}(t)$ of the $m$ th data stream (virtual channel) belonging to the $k$ th user during the $j$ th time interval $I_{j}$ is expressed as

$$
\begin{aligned}
& s_{k m}(t)=\sqrt{2 P} a_{k m}(t) b_{k m}(t) \cos \left(\omega_{c} t+\theta_{k m}\right) \\
& \quad t \in I_{j}, 1 \leq m \leq N_{k j} \leq N_{c}, 1 \leq k \leq K
\end{aligned}
$$

where

$P \quad$ transmission power of the base station;

$\theta_{k m} \quad$ random phase angle, uniformly distributed between 0 and $2 \pi$, introduced by the modulator;

$b_{k m}(t)$ data signal which consists of a sequence of rectangular pulses of duration $T$, i.e.,

$$
b_{k m}(t)=\sum_{i=-\infty}^{\infty} b_{k m}^{(i)} \Pi_{T}(t-i T)
$$


where $b_{k m}^{(i)} \in\{1,-1\}$. The concatenated spreading code $a_{k m}(t)$ can be expressed as

$$
a_{k m}(t)=\sum_{i=-\infty}^{\infty} a_{k m}^{(i)} \Pi_{T_{c}}\left(t-i T_{c}\right)
$$

where $a_{k m}^{(i)} \in\{-1,1\}$ is the concatenated spreading sequence which is equal to the product of a PN sequence $c_{k}^{(i)} \in\{-1,1\}$ used by the $k$ th user and a WH code sequence $d_{m}^{(i)} \in\{-1,1\}$ assigned to its $m$ th virtual channel, i.e.,

$$
a_{k m}^{(i)}=c_{k}^{(i)} \times d_{m}^{(i)}
$$

In (2) and (3), $\Pi_{T}(\cdot)$ is the unit pulse function of duration $T$, defined by

$$
\Pi_{T}(t)= \begin{cases}1, & t \in[0, T) \\ 0, & \text { else }\end{cases}
$$

The duration of each data bit is $T$, while the duration of each chip in the spreading code is $T_{c}$. The number of chips per bit is $N_{c}=T / T_{c}$, where $N_{c}$ is an integer. The period of the $\mathrm{WH}$ code sequence $d_{m}^{(i)}$ is equal to the processing gain $N_{c}$. The long PN sequence $c_{k}^{(i)}$ has a period $N_{\mathrm{PN}}$ that is much greater than $N_{c}$. Moreover, $N_{\mathrm{PN}}$ and $N_{c}$ are chosen to be relatively prime so that every possible chip of the PN sequence can occur at the beginning of some data bit.

As a result, the total signal transmitted to $K$ users is

$$
s(t)=\sum_{k=1}^{K} \sum_{m=1}^{N_{k j}} s_{k m}(t), \quad t \in I_{j}=\bigcap_{k=1}^{K} I_{k j} .
$$

\section{B. Mobile Radio Channel Model}

The Rayleigh multipath fading model is the general accepted channel model for macrocellular mobile communications [10]. In this paper, we adopted the Rayleigh fading model for performance analysis in our multicode CDMA system. The channel impulse response for the $m$ th virtual channel of the $k$ th user is given by

$$
\begin{aligned}
h_{k m}(t)= & \sum_{l=1}^{L_{k m}} \beta_{l k m} \delta\left(t-\tau_{l k m}\right) e^{j \phi_{l k m}} \\
& t \in I_{j}, 1 \leq m \leq N_{k j}, 1 \leq k \leq K
\end{aligned}
$$

where
$\beta_{l k m}$
$l$ th Rayleigh distributed random path gain;
$\phi_{l k m} \quad l$ th random path phase, uniformly distributed be- tween zero and $2 \pi$;
$\tau_{l k m} \quad l$ th uniformly distributed random delay ranging from zero to one data bit period, $T$;
$\delta(t) \quad$ the unit impulse function;
$L_{k m} \quad$ the number of resolvable multipaths for the $m$ th virtual channel of user $k$.

In addition, it should be mentioned that these channel parameters vary with the transmitter-receiver distance. It may be shown that $\bar{\beta}_{l k m}^{2}=\bar{\beta}_{l k 1}^{2}=\bar{\beta}_{l k}^{2}$ and $L_{k m}=L_{k 1}=L_{k}$ for $1 \leq m \leq$ $N_{k j}$, since all the parallel virtual channels introduced by the same user are transmitted over the same propagation environment between the transmitter and receiver and then would have identical channel characteristics, where $\bar{x}^{2}$ denotes the variance of a random variable.

\section{Receiver Model}

The received signal at the input to the matched filter in the mobile receiver is given by

$$
\begin{aligned}
r(t)= & \operatorname{Re}\left\{\int_{-\infty}^{\infty} h_{k m}(\tau) \tilde{s}_{k m}(t-\tau) \cdot \exp \left(j \omega_{c} t\right) d \tau\right\}+n(t) \\
= & \sqrt{2 P} \sum_{k=1}^{K} \sum_{m=1}^{N_{k j}} \sum_{l=1}^{L_{k m}} \beta_{l k m} a_{k m}\left(t-\tau_{l k m}\right) b_{k m}\left(t-\tau_{l k m}\right) \\
& \cdot \cos \left(\omega_{c} t+\varphi_{l k m}\right)+n(t) \\
\varphi_{l k m}= & -\omega_{c} \tau_{l k m}+\phi_{l k m}+\theta_{l k m}
\end{aligned}
$$

where

$\tilde{s}(t) \quad$ complex envelope of $s(t)$;

$\operatorname{Re}\{\cdot\} \quad$ denotes the real part of complex number;

$n(t) \quad$ white Gaussian noise with two-sided power spectral density $N_{0} / 2$.

For simplified analysis, the first virtual channel of the first user is chosen as the reference for calculating the probability of error of its data symbol $b_{11}^{(j)}$ in the $j$ th sampling time interval $I_{s}^{(j)}=[(j-1) T, j T]$. The receiver is able to coherently recover the carrier phase $\varphi_{l k m}$ and $\tau_{l k m}$ locking to the $l$ th path as a reference path between the transmitter of reference and its corresponding receiver. All other paths constitute interference. That is, we assume without loss of generality that $\varphi_{l 11}=0$ and $\tau_{l 11}=0$. The envelope of the matched-filter output at the $j$ th sampling time instant $\left(t=j T\right.$ is denoted by $Y_{11}^{(j)}$ and can be expressed as

$$
\begin{aligned}
& Y_{11}^{(j)}= \int_{(j-1) T}^{j T} r(t) a_{11}(t) \cos \left(\omega_{c} t\right) d t \\
&= \beta_{l 11} \sqrt{\frac{P T^{2}}{2}} b_{11}^{(j)}+\operatorname{Int}_{1}+\operatorname{Int}_{2}+\operatorname{Int}_{3}+\nu \\
& I_{s}^{(j)} \subseteq I_{j}=\bigcap_{k=1}^{K} I_{k j}
\end{aligned}
$$

where

$$
\nu=\int_{(j-1) T}^{j T} n(t) a_{11}(t) \cos \left(\omega_{c} t\right) d t .
$$

Int $_{1}$ intramultiuser interference (self-interference) indicating the interference introduced by the other virtual channels of reference user; 
$\mathrm{Int}_{2} \quad$ intramultipath interference;

$\mathrm{Int}_{3}$ denotes the intermultiuser interference.

$$
\begin{aligned}
\operatorname{Int}_{1}= & \sqrt{P / 2} \sum_{m=2}^{N_{1 j}} \beta_{11 m} b_{1 m}^{(j)} \tilde{R}_{1 m, 11}\left(n_{1}^{\prime}, 0\right) \\
\operatorname{Int}_{2}= & \sqrt{P / 2} \sum_{m=1}^{N_{1 j}} \sum_{\substack{q=1 \\
q \neq l}}^{L_{1 m}}\left\{\beta_{q 1 m} \cos \left(\varphi_{q 1 m}\right)\right. \\
& \times\left[b_{1 m}^{(j-1)} R_{1 m, 11}\left(n_{1}^{\prime}, n_{1}^{\prime}, \tau_{q 1 m}\right)\right. \\
& \left.\left.+b_{1 m}^{(j)} \hat{R}_{1 m, 11}\left(n_{1}^{\prime}, n_{1}^{\prime}, \tau_{q 1 m}\right)\right]\right\}
\end{aligned}
$$

$$
\begin{aligned}
\operatorname{Int}_{3}= & \sqrt{P / 2} \sum_{k=2}^{K} \sum_{m=1}^{N_{k j}} \sum_{q=1}^{L_{k m}}\left\{\beta_{q k m} \cos \left(\varphi_{q k m}\right)\right. \\
& \times\left[b_{k m}^{(j-1)} R_{k m, 11}\left(n_{k}^{\prime}, n_{1}^{\prime}, \tau_{q k m}\right)\right. \\
& \left.\left.+b_{k m}^{(j)} \hat{R}_{k m, 11}\left(n_{k}^{\prime}, n_{1}^{\prime}, \tau_{q k m}\right)\right]\right\}
\end{aligned}
$$

In (11), (12), and (13), $\tilde{R}_{1 m, 11}\left(n_{1}^{\prime}, \tau\right), R_{k m, 11}\left(n_{k}^{\prime}, n_{1}^{\prime}, \tau\right)$, and $\hat{R}_{k m, 11}\left(n_{k}^{\prime}, n_{1}^{\prime}, \tau\right)$ are the well-known continuous periodic cross correlation and partial cross correlation/autocorrelations of the regenerated code and a delayed version of the interfering codes [7], [8], respectively. They are defined as follows:

$$
\begin{aligned}
\tilde{R}_{1 m, 11}\left(n_{1}^{\prime}, \tau\right)= & \int_{(j-1) T}^{j T} a_{1 m}\left(t+n_{1}^{\prime} T_{c}-\tau\right) \\
& \cdot a_{11}\left(t+n_{1}^{\prime} T_{c}\right) d t \\
R_{k m, 11}\left(n_{k}^{\prime}, n_{1}^{\prime}, \tau\right)= & \int_{(j-1) T}^{\tau} a_{k m}\left(t+n_{k}^{\prime} T_{c}-\tau\right) \\
& \cdot a_{11}\left(t+n_{1}^{\prime} T_{c}\right) d t \\
\hat{R}_{k m, 11}\left(n_{k}^{\prime}, n_{1}^{\prime}, \tau\right)= & \int_{\tau}^{j T} a_{k m}\left(t+n_{k}^{\prime} T_{c}-\tau\right) \\
& \cdot a_{11}\left(t+n_{1}^{\prime} T_{c}\right) d t
\end{aligned}
$$

where $n_{k}^{\prime}$ is the initial phase of the PN sequence used by the $k$ th user.

Fong et al. [7], [8] have shown that $\tilde{R}_{1 m, 11}\left(n_{1}^{\prime}, 0\right)$ is always equal to zero when $a_{1 m}(t)$ and $a_{11}(t)$ are the concatenated spreading codes. This implies that the orthogonality of the concatenated spreading codes eliminates the intramultiuser interference (self-interference).

\section{Determination of Maximum Number (Capacity) of WH codes Based on the Quality Requirement of Multimedia Service}

Usually, the bit error rate (BER) can be regarded as the performance index used to evaluate the quality of multimedia object, such as audio, video, and text. For the simplified derivation of the BER, the Gaussian assumption is to take all the self-interference, intramultipath interference, and intermultiuser interference terms as Gaussian noise. To calculate the overall variance of the interference terms, one should evaluate a term like as shown in (17), shown at the bottom of the page.

Pursley [11] showed that $\kappa^{2}$ is a constant which has the value $2 / 3 N_{c}$. Since the interference terms in (9) are all mutual conditionally independent, the overall variance becomes

$$
\sigma^{2}=\operatorname{var}\{\nu\}+\operatorname{var}\left\{\operatorname{Int}_{1}\right\}+\operatorname{var}\left\{\operatorname{Int}_{2}\right\}+\operatorname{var}\left\{\operatorname{Int}_{3}\right\}
$$

where $\operatorname{var}\{\nu\}=N_{0} T / 4$ and $\operatorname{var}\left\{\operatorname{Int}_{1}\right\}=0$. It is shown in Section III-B that $\beta_{l k m}^{2}=\beta_{l k}^{2}$ and $L_{k m}=L_{k}$ for $1 \leq m \leq N_{k j}$ and $1 \leq k \leq K$. Therefore, the expressions of $\operatorname{var}\left\{\operatorname{Int}_{2}\right\}$ and $\operatorname{var}\left\{\operatorname{Int}_{3}\right\}$ are, respectively, given by

$$
\begin{aligned}
& \operatorname{var}\left\{\operatorname{Int}_{2}\right\}=\frac{P T^{2}}{4} \kappa^{2} N_{1 j} \times\left\{\sum_{\substack{q=1 \\
q \neq l}}^{L_{1}} \bar{\beta}_{q 1}^{2}\right\} \\
& \operatorname{var}\left\{\operatorname{Int}_{3}\right\}=\frac{P T^{2}}{4} \kappa^{2} \times\left\{\sum_{k=2}^{K} N_{k j} \sum_{q=1}^{L_{k}} \bar{\beta}_{q k}^{2}\right\} .
\end{aligned}
$$

Moreover, since all the signals including the desired signal and the interfering signals caused by the other $(K-1)$ users relative to the reference user (user 1) are transmitted to the first mobile receiver from the same base station (downlink) and have the identical propagation environment between the base station and the receiver for user 1 , it can be shown that $\bar{\beta}_{q k}^{2}=\bar{\beta}_{q 1}^{2}$ and $L_{k}=L_{1}=L$. From the previous discussion, the variance of the total interfering signals then becomes

$$
\begin{aligned}
\operatorname{var}\left\{\operatorname{Int}_{2}\right\}+\operatorname{var}\left\{\operatorname{Int}_{3}\right\} & =\frac{P T^{2} \kappa^{2}}{4} \times\left\{N_{1 j} \times\left(\sum_{q=1}^{L} \bar{\beta}_{q}^{2}\right)\right. \\
& \left.=\frac{K \neq l}{K} \sum_{k=2} N_{k j} \times\left(\sum_{q=1}^{L} \bar{\beta}_{q}^{2}\right)\right\} \\
& =\frac{P T^{2} \kappa^{2}}{4}\left\{M \times\left(\sum_{q=1}^{L} \bar{\beta}_{q}^{2}\right)-N_{1 j} \bar{\beta}_{l}^{2}\right\} \\
& =\frac{\bar{E}_{b} T \kappa^{2}}{4}\left\{M \times \eta-N_{1 j}\right\}
\end{aligned}
$$

$$
\kappa^{2}=E\left\{\frac{\left[b_{k m}^{(j-1)} R_{k m, 11}\left(n_{k}^{\prime}, n_{1}^{\prime}, \tau_{q k m}\right)+b_{k m}^{(j)} \hat{R}_{k m, 11}\left(n_{k}^{\prime}, n_{k}^{\prime}, \tau_{q k m}\right)\right]^{2}}{T^{2}}\right\}
$$


where $\bar{E}_{b}\left(=\bar{\beta}_{l}^{2} P T\right)$ represents the received signal energy per bit via the $l$ th path (reference path), $M=\sum_{k=1}^{k} N_{k j}$, and $\eta=$ $\sum_{q=1}^{L}\left(\bar{\beta}_{q}^{2} / \bar{\beta}_{l}^{2}\right)$. Similarly, the received signal power is found to be $\left(T \bar{E}_{b} / 2\right)\left(=\left(P T^{2} \bar{\beta}_{l}^{2}\right) / 2\right)$. Hence, the average value of half the signal-to-noise plus interference power ratio becomes

$$
\begin{aligned}
\bar{\gamma}_{b} & =\frac{1}{2} \frac{\frac{T \bar{E}_{b}}{2}}{\frac{T \bar{E}_{b} \kappa^{2} M}{4} \times\left[\eta-\frac{N_{1 j}}{M}\right]+\frac{N_{0} T}{4}} \\
& =\left[\frac{2 M}{3 N_{c}}\left(\eta-\frac{N_{1 j}}{M}\right)+\frac{N_{0}}{\bar{E}_{b}}\right]^{-1} .
\end{aligned}
$$

In addition, assume that $\eta \times M \gg N_{i j}$. Then, $\bar{\gamma}_{b}$ becomes

$$
\bar{\gamma}_{b} \approx\left[\frac{2 M \eta}{3 N_{c}}+\frac{N_{0}}{\bar{E}_{b}}\right]^{-1}
$$

Note that $\eta$ is always greater than one. For simplicity, assume that the path gains for all the virtual channels in the same user are independently identically distributed (i.i.d.), i.e., $\bar{\beta}_{q}^{2}=\bar{\beta}^{2}$, $1 \leq q \leq L$. Thus, the value of $\eta$ is identical to $L$, i.e., $\eta=L$, and the expression of (23) for large $L M$ reduces to

$$
\bar{\gamma}_{b} \approx\left[\frac{2 M L}{3 N_{c}}+N_{0} / \bar{E}_{b}\right]^{-1}
$$

Moreover, for the application of multimedia presentation services, the interference term can be reduced by a multimedia presentation activity factor of $\alpha$ [6] given by

$$
\alpha=f_{\text {video }} \times \alpha_{\text {video }}+f_{\text {audio }} \times \alpha_{\text {audio }}+f_{\text {text }} \times \alpha_{\text {text }}
$$

where $\alpha_{O}$ and $f_{O}$ are the activity factor and the presentation occurrence frequency for object $O$, respectively. Thus, with monitoring multimedia presentation activity, $\bar{\gamma}_{b}$ is increased relative to (24)and becomes

$$
\bar{\gamma}_{b} \approx\left[\frac{2 M L}{3 N_{c}} \alpha+N_{0} / \bar{E}_{b}\right]^{-1} .
$$

Proakis [10] showed that the BER for both the nondiversity coherent receiver and a receiver with maximal ratio combining (MRC) of order $L_{d}$ can be expressed as a form in terms of $\bar{\gamma}_{b}$

$$
\begin{aligned}
& p_{e}=p_{e}\left(\bar{\gamma}_{b}\right) \\
& =\left\{\begin{array}{cl}
\frac{1}{2}\left[1-\sqrt{\frac{\bar{\gamma}_{b}}{1+\bar{\gamma}_{b}}}\right], & \text { for nondiversity receiver } \\
\left(\frac{1-\mu}{2}\right)^{L_{d}} \times \sum_{s=0}^{L d-1} & \\
\times\left(\begin{array}{c}
L_{d}-1+s \\
s
\end{array}\right) & \\
\times\left(\frac{1+\mu}{2}\right)^{s}, & \text { for MRC of order } L_{d} .
\end{array}\right.
\end{aligned}
$$

In (27), for MRC, the quantity $\bar{\gamma}_{b}$ represents the average signal-to-noise ratio (SNR) per combined path, and $\mu=\sqrt{\bar{\gamma}_{b} /\left(1+\bar{\gamma}_{b}\right)}$.

Assume that $\mathrm{BER}_{\text {mult }}$ denotes the BER requirement for supporting the acceptable quality of multimedia presentation ser- vice. The value of $B E R_{\text {mult }}$ (in $\mathrm{dB}$ ) can be obtained by calculating the weighted sum of $\mathrm{BER}_{\text {video }}$ (in $\mathrm{dB}$ ), $\mathrm{BER}_{\text {audio }}$ (in $\mathrm{dB}$ ), and $\mathrm{BER}_{\text {text }}$ (in $\mathrm{dB}$ ) and is given by

$$
\begin{aligned}
\mathrm{BER}_{\text {mult }}(\mathrm{dB})= & w_{v} \mathrm{BER}_{\text {video }}(\mathrm{dB})+w_{a} \mathrm{BER}_{\text {audio }}(\mathrm{dB}) \\
& +w_{t} \mathrm{BER}_{\text {text }}(\mathrm{dB})
\end{aligned}
$$

where $\mathrm{BER}_{O}$ and $w_{O}$ are the BER requirement and weighting factor for object $O$, respectively, where $\mathrm{BER}_{O}=10 \log _{10} p_{e}\left(\bar{\gamma}_{b}\right)(\mathrm{dB})$. Note that the logarithmic scale is able to make $\mathrm{BER}_{\text {video }}, \mathrm{BER}_{\text {audio }}$, and $\mathrm{BER}_{\text {text }}$ be in the same order of magnitude. Thus, the maximum number of $\mathrm{WH}$ codes assigned to all the multimedia users during the time interval $I_{j}$ is determined by

$$
M_{\max }=\frac{3 N_{c}}{2 \alpha L}\left[\frac{1}{p_{e}^{-1}\left\{\exp \left(\frac{\mathrm{BER}_{\text {mult }}}{10}\right)\right\}}-N_{0} / \bar{E}_{b}\right]
$$

where $p_{e}^{-1}(\cdot)$ denotes the inverse function of $p_{e}(\cdot)$ which is a monotonically decreasing function of $\mathrm{BER}_{\text {mult }}$. Equation (29) shows that $M_{\max }$ is increasing when $\mathrm{BER}_{\text {mult }}$ is increasing or the degree of multimedia presentation quality is decreasing. However, it should be noted that $M_{\max }$ of (29) is used to support the presentation quality of OCPN belonging to the reference user (user 1). In order to maintain the presentation quality of BER mult for all the $K$ OCPN's, $M_{\max }$ of (29) should be selected as follows:

$$
\begin{aligned}
& M_{\max }=\operatorname{Min}_{1 \leq k \leq K}\left\{M_{\max }^{(k)}\right\} \\
& =\frac{3 N_{c}}{2 \alpha_{\max } L_{\max }}\left[\frac{1}{p_{e}^{-1}\left\{\exp \left(\frac{\mathrm{BER}_{\text {mult }}}{10}\right)\right\}}-N_{0} / \bar{E}_{b, \min }\right]
\end{aligned}
$$

where

$M_{\max }^{(k)} \quad$ maximum number of WH codes assigned to user $k$;

$\exp \{\cdot\} \quad$ exponential function with a base of ten.

In other words, $\exp \{x\}=10^{x}, \alpha_{\max }=\max _{1 \leq k \leq K}\left[\alpha^{(k)}\right]$, $L_{\max }=\max _{1 \leq k \leq K}\left[L^{(k)}\right]$, and $\bar{E}_{b, \min }=\min _{1 \leq k \leq K}\left[\bar{E}_{b}^{(k)}\right]$. $\alpha^{(k)}$ denotes the presentation activity factor of the $k$ th OCPN. $L^{(k)}$ and $\bar{E}_{b}^{(k)}$ represent the number of multipaths and the received signal energy per bit from the base station to the $k$ th mobile receiver, respectively. Therefore, the quality of all the $K$ OCPN's may satisfy the BER requirement. In the near future, we will propose a new power assignment technique to select an appropriate level of transmitting power for each user in order to maximize the value of (30). For this case, the transmitting power for user $k$ of (1) may be different and can be selected to achieve the maximum capacity.

\section{Dynamic SpReading Code ASSIGNMENT FOR OCPN-BASED MUlTimedia SERVICES}

The base station is responsible for assigning the appropriate number of WH codes for every multimedia object in all the concurrent OCPN's in each time interval. It is therefore essential 
that information about OCPN's, i.e., the playout deadline $\pi_{i}$, the playout duration $\tau_{i}$, and the throughput requirement $\left|O_{i}\right|$ of object $O_{i}$ in the OCPN's must be provided to the base station at the time of connection establishment prior to assigning WH codes (or virtual channels) to those objects. When the total number requirement of WH codes assigned to all the OCPN's is greater than $M_{\max }$ of (30), and the total number of WH codes assigned to each OCPN is also less than $N_{c}$, the transmission rate of multimedia objects over the mobile radio channel can be matched with the playout rate at the mobile terminal, and the presentation quality of each OCPN is guaranteed with a BER value less than BER $_{\text {mult }}$ of (28). However, the maximum number of $\mathrm{WH}$ codes may not be sufficient to accommodate all the OCPN's for a certain time interval. In this case, reassignment of $\mathrm{WH}$ codes is needed at the occurrence of transitions in OCPN's. The possible way to code reassignment is based on either dropping some SIU's in order to decrease total number requirement of WH codes assigned to all the OCPN's or increasing the value of $\mathrm{BER}_{\text {mult }}$ in order to increase to value of $M_{\max }$. Both strategies result in the degradation in presentation quality. Woo et al. [4] have shown that the tolerable loss of SIU's can be expressed in terms of reliability requirement $\zeta$. For example, $\zeta$ audio $=0.98$, $\zeta_{\text {video }}=0.9, \zeta_{\text {text }}=1$. In other words, the presentation of object $O$ with loss of $(1-\zeta) \%$ information is tolerable.

We can define the loss of information as the "dropping ratio" $\theta_{i}$ of object $O_{i}$. It represents the degradation in the transmission of object $O_{i}$ as a result of that the code number requirement for all the users exceeds the maximum number of available WH codes. As an example, for a video object whose code number requirement is less than a given $M_{\max }$, the number of video frames has been dropped with a ratio of $\theta \%$ in order to reduce the code number requirement. However, the quality of each survived video frame is still maintained with a constant performance of $\mathrm{BER}_{\text {mult }}$ during the presentation period. In terms of SIU's of object $O_{i}$, the ratio $\theta_{i}$ is given as

$$
\theta_{i}=\frac{\text { number of SIU's dropped in } O_{i}}{\text { Total number of SIU's in } O_{i}} .
$$

Woo et al. [4] indicated that expressing the ratio $\theta_{i}$ in terms of SIU's provides a better control mechanism for distributing the total droppage of object $O_{i}$ uniformly over all its SIU's.

\section{A. Spreading Code Assignment for Concurrent Multimedia Objects in A Fixed Time Interval}

A fair code assignment policy requires that the degradation should be evenly spread across all the objects that are being transmitted concurrently if transmission needs to be degraded. The degradation in transmission occurs when either code number requirement for all the OCPN's (users) or code number requirement for each OCPN (user) exceeds its maximum available code number in time interval $I$. The previous argument implies that all the objects have roughly equal dropping ratios. Moreover, all the dropping ratio $\theta_{i}^{\prime} s$ should be selected to minimize the total degradation loss of all the OCPN's. Under the earlier discussions, nonlinear programming (NLP) [12] is proposed to find the dropping ratio $\theta_{i}^{\prime} s$ for objects $O_{i}^{\prime} s \in \psi$ in an interval $I$ to conform the limited available number of $\mathrm{WH}$ codes, where $\psi$ is a set of all the concurrent objects in an interval $I$. Prior to describing the procedure for code assignment, notations used in the procedure are defined as follows: $\left|O_{i}\right|$ denotes the throughput requirement of object $O_{i}, r_{B}$ represents the basic transmission bit rate for the multicode CDMA system, $\psi_{k}$ is the set of all the concurrent objects belonging to the $k$ th user in an interval $I$, and $N$ and $N^{(k)}$ are the code number requirements in an interval $I$ for all the $K$ users and the $k$ th user, respectively. They are defined as: $N=\sum_{O_{i} \in \psi}\left\lceil\left|O_{i}\right| / r_{B}\right\rceil$ and $N^{(k)}=\sum_{O_{i} \in \psi_{k}}\left\lceil\left|O_{i}\right| / r_{B}\right\rceil$.

A procedure for code assignment in an interval $I$ is described as follows.

Step 1) Satisfaction for all the code number requirements.

a) If $N \leq M_{\max }$ and $N^{(k)} \leq N_{c}, 1 \leq k \leq K$, then perform the following procedure. Otherwise, go to Step 2.

b) Code assignment:

$n_{i}=$ number of WH codes assigned to object $O_{i}$

$=\left\lceil\left|O_{i}\right| / r_{B}\right\rceil, \quad O_{i} \in \psi$.

c) Terminate the procedure, and then output $n_{i}^{\prime} s$.

Step 2) Violation for the code number requirement per user.

a) If $N \leq M_{\max }$ and there exists a user, i.e., user $k$ such that $N^{(k)}>N_{c}$, then perform the following procedure. Otherwise, go to Step 3.

b) Drop some SIU's of objects $O_{i}$ in $\psi_{k}$ such that $N^{(k)}=N_{c}$. Solve the NLP:

$$
\begin{aligned}
& \text { NLP1: Minimize } E^{(k)}=w_{1} E_{1}^{(k)}+w_{2} E_{2}^{(k)} \\
& \text { subect to } \\
& \text { i) } \\
& \qquad \sum_{O_{i} \in \psi_{k}}\left\lceil\left(1-\theta_{i}\right)\left|O_{i}\right| / r_{B}\right\rceil=N_{c}
\end{aligned}
$$

ii) Search range:

$$
0 \leq \theta_{i} \leq 1-\zeta_{i}, O_{i} \in \psi_{k}
$$

where $E_{1}^{(k)}$ and $E_{2}^{(k)}$ are the costs used to measure the uniformity of $\theta_{i}^{\prime} s$ and the degradation loss for object $k$, respectively. They are defined as follows:

$$
\begin{aligned}
& E_{1}^{(k)}=\sum_{\substack{O_{i}, O_{l} \in \psi_{k} \\
i<l}}\left(\theta_{i}-\theta_{l}\right)^{2} \\
& E_{2}^{(k)}=\sum_{O_{i} \in \psi_{k}} \theta_{i}^{2}
\end{aligned}
$$

$w_{1}$ and $w_{2}$ are their associated weighting factors.

c) Find the optimal solution $\theta_{i}^{*}$ 's, $O_{i} \in \psi_{k}$ to NLP1 of (33). Thus, their code assignment is

$$
n_{i}= \begin{cases}\left\lceil\left(1-\theta_{i}^{*}\right)\left|O_{i}\right| / r_{B}\right\rceil & \text { if } O_{i} \in \psi_{k} \\ \left\lceil\left|O_{i}\right| / r_{B}\right\rceil & \text { if } O_{i} \notin \psi_{k} .\end{cases}
$$

d) Terminate the procedure, and then output $n_{i}^{\prime} s$. 
Step 3) Violation for the code number requirement for total $K$ users

a) If $N>M_{\max }$, then perform the following procedure

b) NLP2:

$\operatorname{Min} E=w_{1} E_{1}+w_{2} E_{2}$

subject to

$$
\sum_{O_{i} \in \psi}^{\text {i) }}\left\lceil\left(1-\theta_{i}\right)\left|O_{i}\right| / r_{B}\right\rceil=M_{\max }
$$

ii)

$$
\sum_{O_{i} \in \psi_{k}}\left\lceil\left(1-\theta_{i}\right)\left|O_{i}\right| / r_{B}\right\rceil \leq N_{c}
$$

iii) Search range:

$$
0 \leq \theta_{i} \leq 1-\zeta_{i}, O_{i} \in \psi
$$

where

$$
E_{1}=\sum_{\substack{O_{i}, O_{l} \in \psi \\ i<l}}\left(\theta_{i}-\theta_{l}\right)^{2}
$$

and

$$
E_{2}=\sum_{O_{i} \in \psi} \theta_{i}^{2}
$$

denote the measure of uniformity and the degradation loss for total $K$ users, respectively.

c) Find the optimal solution $\theta_{i}^{*}$ 's, $O_{i} \in \psi$ to NLP2 of (39). Thus, their code assignment is

$$
n_{i}=\left\lceil\left(1-\theta_{i}^{*}\right)\left|O_{i}\right| / r_{B}\right\rceil, \quad O_{i} \in \psi
$$

d) Terminate the procedure, and output $n_{i}^{\prime} s$.

NLP1 or NLP2, which are indeed quadratic programming problems, can be solved efficiently by a variety of techniques [12]. However, it is quite plausible that the solution to the aforementioned NLP1 or NLP2 is not feasible. If no solution exists, then the reliability requirement $\zeta$ of an object $O$ cannot be accommodated with the given maximum available number of WH codes $M_{\max }$. The feasible solution to NLP1 or NLP2 can be achieved by either decreasing the reliability requirement or increasing the value of $M_{\max }$. This implies that the search range of (35) or (42) becomes larger or the desired quality of BER $_{\text {mult }}$ is degraded. For the case of decreasing $\zeta$ in the video application with a given $M_{\max }$, the maximum dropping ratio $(1-\zeta) \%$ of video frame is increasing. However, the quality of each video frame is still maintained with a constant performance of $\mathrm{BER}_{\text {mult }}$ during the presentation period.

\section{B. Dynamic Spreading Code Assignment}

The aforementioned code assignment is valid for all the concurrent objects that start their presentation at a time instant in a fixed time interval. However, for a general OCPN-based multimedia presentation, some objects may start their presentations at a time instant in the previous time interval. Therefore, the earlier code assignment should be modified to deal with the general multimedia presentation. Similarly, prior to describing the

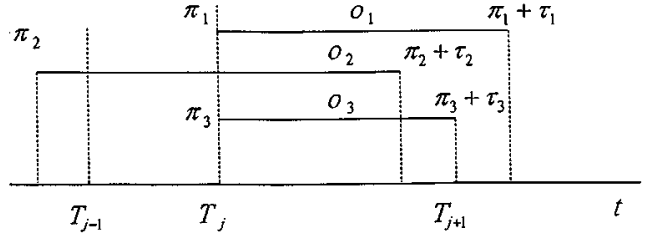

Fig. 4. Illustration of the time relationship between $O_{1}, O_{2}$, and $O_{3}: \Psi_{j}^{s}$ and $\Psi_{j}^{c} ; O_{1}, O_{3} \in \Psi_{j}^{s}$, and $O_{2} \in \Psi_{j}^{c}$.

dynamic code assignment, some notations and parameters used in the assignment are defined. Let $I_{j}$ denote the $j$ th interval $\left[T_{j}, T_{j+1}\right)$ as the current time interval. $\psi_{j}$ denotes the set of concurrent objects in $I_{j}$, which is composed of two disjoint subsets, $\psi_{j}^{s}$ and $\psi_{j}^{c}$. $\psi_{j}^{s}$ represents a set of new concurrent objects that start their presentation at $T_{j} . \psi_{j}^{c}$ consists of objects that continue their presentations during interval $I_{j}$. $\psi_{k j}$ denotes the set of objects belonging to $\psi_{j}$ for user $k$. Similarly, $\psi_{k j}^{s}$ and $\psi_{k j}^{c}$ represent the sets of objects belonging to $\psi_{j}^{s}$ and $\psi_{j}^{c}$ for user $k$, respectively. Formally, $\psi_{j}\left(\right.$ or $\left.\psi_{k j}\right), \psi_{j}^{s}\left(\right.$ or $\left.\psi_{k j}^{s}\right)$, and $\psi_{j}^{c}\left(\right.$ or $\left.\psi_{k j}^{c}\right)$ can be defined as follows:

$$
O_{i} \in \begin{cases}\psi_{j}^{s}\left(\text { or } \psi_{k j}^{s}\right), & \text { if } \pi_{i}=T_{j} \\ \psi_{j}^{c}\left(\text { or } \psi_{k j}^{c}\right), & \text { if } \pi_{i}<T_{j}<\pi_{j}+\tau_{j} .\end{cases}
$$

Fig. 4 illustrates an example of $O_{1}, O_{3} \in \psi_{j}^{s}$, and $O_{2} \in \psi_{j}^{c}$.

$M_{a}(t)$ denotes the available number of WH codes assigned to all the $K$ users at time $t$. Similarly, let $M_{a}^{(k)}(t)$ be the available number of WH codes assigned to user $k$ at time $t, 1 \leq k \leq K$. $n_{i j}$ represents the total number of WH codes assigned to object $O_{i}$ belonging to $\psi_{j}$ in $I_{j} . N_{j}^{s}$ and $N_{k j}^{s}$ are the code number requirements for new concurrent objects belonging to $\psi_{j}^{s}$ and $\psi_{k j}^{s}$ in $I_{j}$, respectively. Let $T_{j}^{-}$denote the time just before the transition at time $T_{j}$ is fired. Thus, the available number of $\mathrm{WH}$ codes before firing the transition at $T_{j}$ is computed by

$$
\begin{aligned}
M_{a}\left(T_{j}^{-}\right)= & M_{a}\left(T_{j-1}\right)+(\text { total number of WH codes } \\
& \text { released by all the objects ended at a time } \\
& \text { instant between } \left.T_{j-1} \text { and } T_{j}\right) \\
= & M_{a}\left(T_{j-1}\right)+\sum_{\left\{O_{i} \mid \pi_{i}+\tau_{i} \in I_{j-1}\right\}} n_{i, j-1} .
\end{aligned}
$$

Note that the expression of (45) is also valid for $M_{a}^{(k)}\left(T_{j}^{-}\right)$, $1 \leq k \leq K$. The base station should update its available code number at the occurrence of each transition, and it is also able to assess the code number requirements for the next interval, based on the information given in OCPN's of the connected users. In other words, the code assignment for the next interval $I_{j+1}$ can be determined continuously ahead of time by inspecting code number requirements specified in OCPN's.

The procedure for dynamic code assignment is summarized as follows.

Step 0) Initialization: $J=$ maximum time index, $M_{a}\left(T_{0}^{-}\right)=$ $M_{\max }, M_{a}^{(k)}\left(T_{0}^{-}\right)=N_{c}, 1 \leq k \leq K, n_{i,-1}=$ $0 \forall i$, and $j=0$.

Step 1) Test the termination:

If $j>J$, then terminate the procedure.

Otherwise, go to Step 2. 
Step 2) Code number requirements for new objects:

$$
N_{j}^{s}=\sum_{O_{i} \in \psi_{j}^{s}}\left\lceil\left|O_{i}\right| / r_{B}\right\rceil
$$

and

$$
N_{k j}^{s}=\sum_{O_{i} \in \psi_{k j}^{s}}\left\lceil\left|O_{i}\right| / r_{B}\right\rceil, \quad 1 \leq k \leq K
$$

Step 3) Satisfaction for all the code assignment requirements: if $N_{j}^{s} \leq M_{a}\left(T_{j}^{-}\right)$and $N_{k j} \leq M_{a}^{(k)}\left(T_{j}^{-}\right), 1 \leq k \leq$ $K$, then perform the following procedure. Otherwise, go to Step 4.

a) Code assignment:

$$
n_{i j}= \begin{cases}\left\lceil\left|O_{i}\right| / r_{B}\right\rceil, & \text { if } O_{i} \in \psi_{j}^{s} \\ n_{i, j-1}, & \text { if } O_{i} \in \psi_{j}^{c} .\end{cases}
$$

b) After code assignment for the new objects, the available code number at $T_{j}$ becomes

$$
\begin{aligned}
M_{a}\left(T_{j}\right) & =M_{a}\left(T_{j}^{-}\right)-\sum_{O_{i} \in \psi_{j}^{s}} n_{i j} \\
M_{a}^{(k)}\left(T_{j}\right) & =M_{a}^{(k)}\left(T_{j}^{-}\right)-\sum_{O_{i} \in \psi_{j k}^{s}} n_{i j}, 1 \leq k \leq K .
\end{aligned}
$$

and then go to Step 6 .

Step 4) Violation for code number requirement per user:

a) If $N^{s} \leq M_{a}\left(T_{j}^{-}\right)$, and there exists a user, i.e., user $k$, such that $N_{k j}^{s}>M_{a}^{(k)}\left(T_{j}^{-}\right)$, then perform the following procedure. Otherwise, go to Step 5.

b) NLP1:

$\operatorname{Min} E_{s}^{(k)}=w_{1} E_{s 1}^{(k)}+w_{2} E_{s 2}^{(k)}, \quad O_{i} \in \psi_{k j}^{s}$ subject to

i) $\sum_{O_{i} \in \psi_{k j}^{s}}\left\lceil\left(1-\theta_{i}\right)\left|O_{i}\right| / r_{B}\right\rceil=M_{a}^{(k)}\left(T_{j}^{-}\right)$

ii) $0 \leq \theta_{i} \leq 1-\zeta_{i}, \quad O_{i} \in \psi_{k}$

where

$$
E_{s 1}^{(h)}=\sum_{\substack{O_{i}, O_{l} \in \psi_{k j}^{s} \\ i<l}}\left(\theta_{i}-\theta_{l}\right)^{2}
$$

and

$$
E_{s_{2}}^{(k)}=\sum_{O_{i} \in \psi_{k j}^{s}} \theta_{i}^{2}
$$

c) Find the optimal solution $\theta_{i}^{*}$ 's, $O_{i} \in \psi_{k j}^{s}$ to NLP1 of (48), and their associated code assignment is given as

$$
n_{i j}= \begin{cases}\left\lceil\left(1-\theta_{i}^{*}\right)\left|O_{i}\right| / r_{B}\right\rceil, & \text { if } O_{i} \in \psi_{k j}^{s} \\ \left\lceil\left|O_{i}\right| / r_{B}\right\rceil, & \text { and user } k \text { has violation } \\ & \text { if } O_{i} \in \psi_{k j}^{s} \\ n_{i, j-1}, & \text { and user } k \text { has satisfaction } \\ \text { if } O_{i} \in \psi_{k j}^{c} .\end{cases}
$$

d)

$$
\begin{aligned}
M_{a}\left(T_{j}\right)= & M_{a}\left(T_{j}^{-}\right)-\sum_{O_{i} \in \psi_{j}^{s}} n_{i j} \\
M_{a}^{(k)}\left(T_{j}\right)= & M_{a}^{(k)}\left(T_{j}^{-}\right)-\sum_{O_{i} \in \psi_{j k}^{s}} n_{i j} \quad 1 \leq k \leq K \\
& \text { and then go to Step } 6 .
\end{aligned}
$$

Step 5) Violation for code number requirement for total $K$ users.

a) If $N_{j}^{s}>M_{a}\left(T_{j}^{-}\right)$, then perform the following procedure:

b) NLP2:

$\operatorname{Min} E_{s}=w_{1} E_{s 1}+w_{2} E_{s 2}, \quad O_{i} \in \psi_{j}^{s}$ subject to

i) $\sum_{O_{i} \in \psi_{j}^{s}}\left\lceil\left(1-\theta_{i}\right)\left|O_{i}\right| / r_{B}\right\rceil=M_{a}\left(T_{j}^{-}\right)$
ii) $\sum_{O_{i} \in \psi_{k j}^{s}}\left\lceil\left(1-\theta_{i}\right)\left|O_{i}\right| / r_{B}\right\rceil \leq M_{a}^{(k)}\left(T_{j}^{-}\right)$

iii) $0 \leq \theta_{i} \leq 1-\zeta_{i}$

where

$$
E_{s 1}=\sum_{\substack{O_{i}, O_{l} \in \psi_{j}^{s} \\ i<l}}\left(\theta_{i}-\theta_{l}\right)^{2}
$$

and

$$
E_{s 2}=\sum_{O_{i} \in \psi_{j}^{s}} \theta_{i}^{2} .
$$

c) Find the optimal solution $\theta_{i}^{*}$ 's, $O_{i} \in \psi_{j}^{s}$ to NLP2 of (50), and their associated code assignment is given as

$n_{i j}=\left\{\begin{array}{l}\left\lceil\left(1-\theta_{i}^{*}\right)\left|O_{i}\right| / r_{B}\right\rceil \\ n_{i, j-1} .\end{array} \quad\right.$ if $O_{i} \in \psi_{j}^{c}$

d)

$$
\begin{aligned}
M_{a}\left(T_{j}\right) & =M_{a}\left(T_{j}^{-}\right)-\sum_{O_{i} \in \psi_{j}^{s}} n_{i j} \\
M_{a}^{(k)}\left(T_{j}\right) & =M_{a}^{(k)}\left(T_{j}^{-}\right)-\sum_{O_{i} \in \psi_{j k}^{s}} n_{i j} \quad 1 \leq k \leq K
\end{aligned}
$$

and then go to Step 6.

Step 6) Update the available code number for the next time interval.

a) $M_{a}\left(T_{j+1}^{-}\right)=M_{a}\left(T_{j}\right)+\sum_{\left\{O_{i} \mid \pi_{i}+\tau_{i} \in I_{j}\right\}} n_{i j}$

b) $M_{a}^{(k)}\left(T_{j+1}^{-}\right)=M_{a}^{(k)}\left(T_{j}\right)$

$$
+\sum_{\left\{O_{i} \mid \pi_{i}+\tau_{i} \in I_{j}, O_{i} \in \psi_{k}\right\}} n_{i j}, \quad 1 \leq k \leq K
$$

c) $j \leftarrow j+1$, and then go to Step 1 .

\section{ILLUSTRATED EXAMPLE}

Using the previous spreading code assignment procedure, the base station can assign the available WH codes to users dynamically based on information in users' OCPN's and the availability of the station's resources. The channel model described 


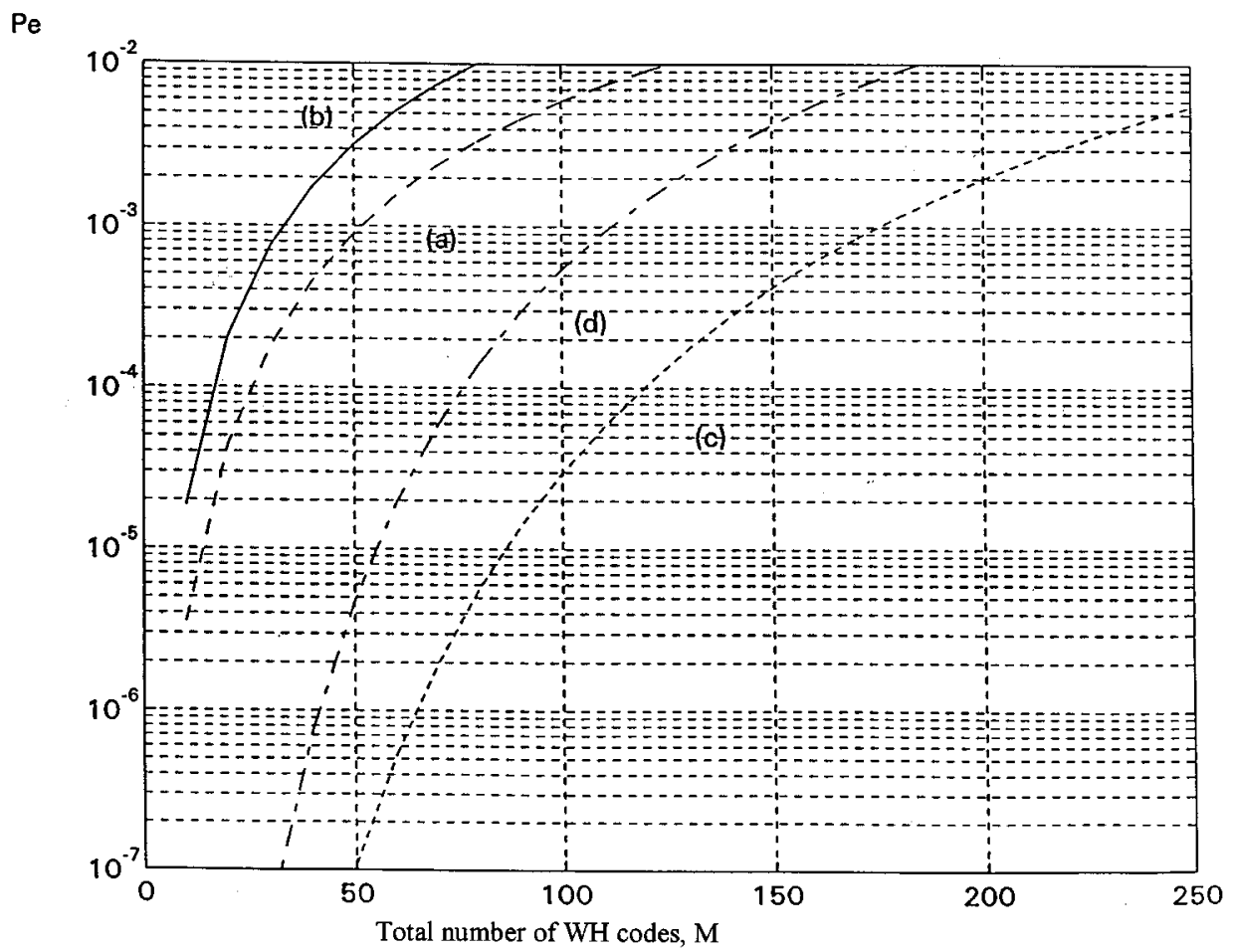

Fig. 5. BER $\left(p_{e}\right)$ versus total number of WH codes assigned to all the $K$ users, $M$. (a) [(b)] and (c) [(d)] denote curves obtained from the MRC and MRC plus $\mathrm{BCH}(15,7)$ coding for the first (second) multimedia title, respectively, where $k=2$.

in this paper is identical to Model-I employed in [13]. In this model, the variances of the Rayleigh path gains are the same for all users and equals $-14 \mathrm{~dB}(0.038)$. Additionally, the maximum number of resolved multipaths for all users is equal to four, i.e., $L=4$. The multicode CDMA system has a transmission rate of $r_{B}=64 \mathrm{~kb} / \mathrm{s}$, a processing gain of $N_{c}=128$, and a bandwidth of $10 \mathrm{MHz}$. Its corresponding PN and orthogonal spreading sequences are chosen as the $\mathrm{m}$-sequence with a period equal to (242-1) and the WH code with a period of 128 , respectively. For the CDMA receiver, it has a maximum ratio combiner (MRC) of order $L_{d}=4$. To evaluate the performance of the multimedia transmission via multicode CDMA channels, two OCPN's for users 1 and 2 are assumed to have two object types, i.e., voice and video objects. For the application of a wireless channel, Khansari et al. [14] showed that voice with source rate equal to $8 \mathrm{~kb} / \mathrm{s}$ and low resolution video with source rate equal to $128 \mathrm{~kb} / \mathrm{s}$ are suitable for such applications. The BER requirements for voice and video are $10^{-3}$ and $10^{-4}$, respectively. From (28), the value of $B R_{\text {mult }}$ is found to be $10^{-3.5}$ or $-35 \mathrm{~dB}$ when $w_{\text {voice }}=w_{\text {video }}=0.5$. Moreover, two different multimedia titles (programs) are conducted to evaluate our system. The first multimedia title has two video and voice object types of identical presentation activity factor. This yields the application of video telephone when $\alpha_{\text {voice }}=\alpha_{\text {video }}=3 / 8$ and $f_{\text {voice }}=f_{\text {video }}=0.5$. For simplicity, here, we assume that $N_{0} / \bar{E}_{b}$ is negligible compared to the multiuser and multipath interference. Thus, from curve (a) of Fig. 5, the maximum number of WH codes is found to be about $M_{\max }^{(1)}=41$ for the first title. The second multimedia title has a higher video presentation activity factor $\alpha_{\text {video }}=0.8, \alpha_{\text {voice }}=3 / 8$, and $f_{\text {voice }}=f_{\text {video }}=0.5$. Curve (b) of Fig. 5 shows that $M_{\max }^{(2)}$ becomes smaller and equals about 29for the second title. By employing the $\mathrm{BCH}(15,7)$ error correction with a code rate of $R_{c}=7 / 15$, curves (c) and (d) show that $M_{\max }$ for both cases become extremely large, i.e., $M_{\max }^{(1)}=150$ for title 1 (video phone) and $M_{\max }^{(2)}=96$ for title 2 . However, the source rates for both video and voice are also increased by a factor of $1 / R_{c}=15 / 7(=2.143)$. This would degrade the transmission efficiency. Meanwhile, it is able to offer multimedia services with lower transmission error via wireless channels.

It should be mentioned that the throughput requirement, $|O|$ of an object $O$ is always set to a multiple of its source rate of $r_{0}$, i.e., $|O|=m \times r_{0}, m \geq 1$, in order to shorten the transmission delay. In other words, a larger number of WH codes are required to provide a sufficient number of virtual channels to meet the throughput requirement. Once the value of $M_{\max }$ has been determined, Fig. 6 illustrates a result of the dynamic code assignment for the two OCPN's in video phone application with $\mathrm{BCH}(15,7)$ channel coding according to $M_{\max }=96=$ $\operatorname{Min}\{150,96\}$ of (30). Fig. 6(a) shows the timeline diagram of the OCPN representations for both users. The first OCPN has three objects $O_{1}, O_{2}$, and $O_{6}$ whereas the second OCPN has four objects $O_{3}, O_{4}, O_{5}$, and $O_{7}$. Note that each of those objects belongs to either video or voice type and has its corresponding throughput requirement. For example, the throughput requirement of $O_{3}$ is almost twice more than that of $O_{1}$ or $O_{2}$. Multimedia objects $O_{1}, O_{2}$, and $O_{3}$ start their presentation at time $T_{0}$ simultaneously. Prior to the presentation, the base station should perform the proposed code assignment procedure to determine the appropriate number of WH codes for each object. Fig. 6(b) 

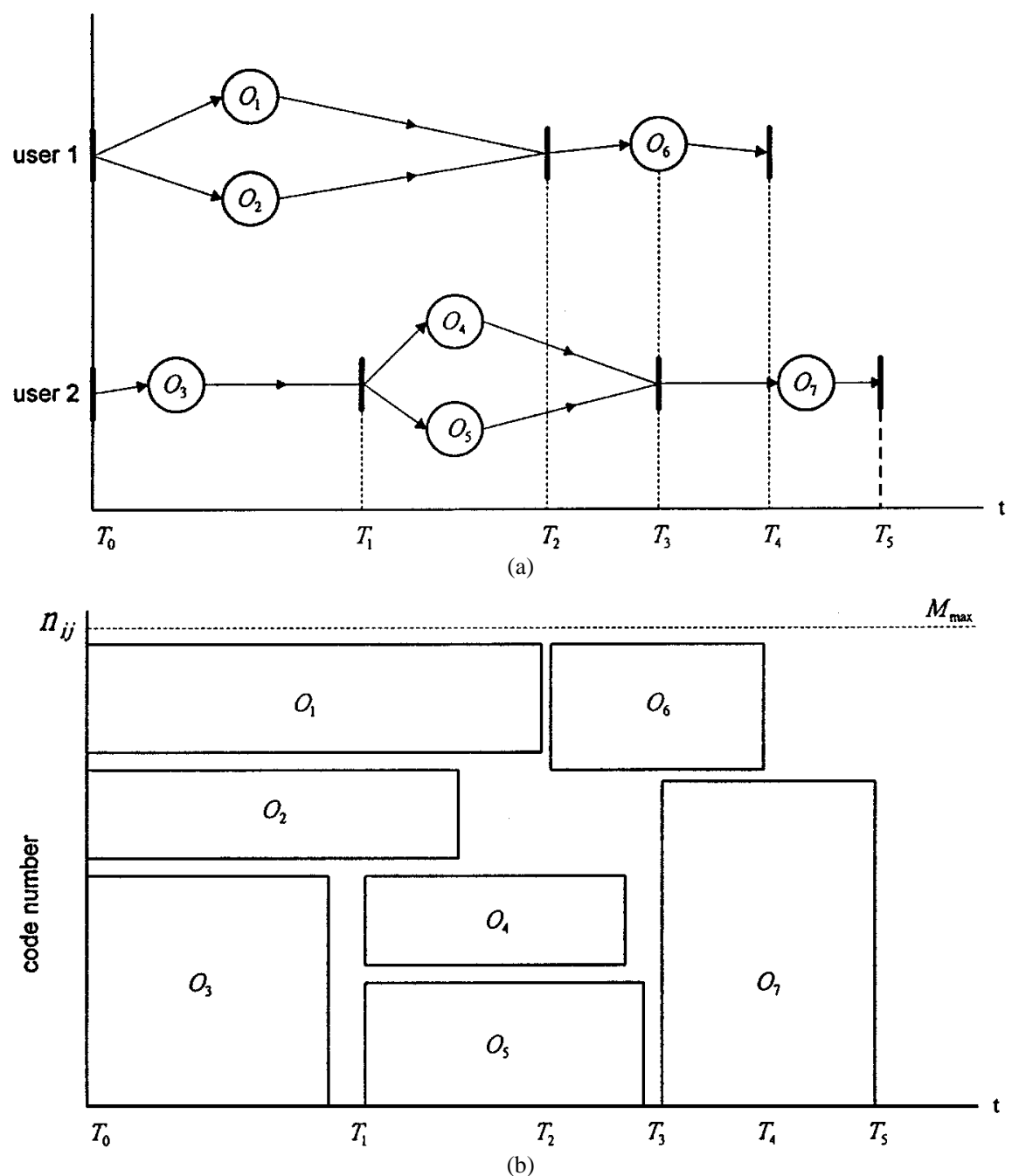

Fig. 6. (a) Example of OCPN's for users 1 and 2. (b) Dynamic code assignment for OCPN-based multimedia objects, where $n_{i j}$ denotes the WH code number assigned to $O_{i}$ for the $j$ th time interval $\left[T_{j}, T_{j+1}\right)$ and $M_{\max }=96$.

shows the resulting code number assigned to $O_{i}$ for the $j$ th time interval $\left[T_{j}, T_{j+1}\right)$, denoted by $n_{i j}$. At time $T_{0}^{-}$, it is found that there are $n_{10}(=26), n_{20}(=22)$ and $n_{30}(=48)$, for $\left[T_{0}, T_{1}\right)$ where $n_{10}+n_{20}+n_{30} \leq M_{\max }=96, n_{10}+n_{20} \leq N_{c}=128$, and $n_{30} \leq 128$. Thus, $M_{a}\left(T_{0}\right)=96-26-22-48=0$ For the next time interval $\left[T_{1}, T_{2}\right)$, when a new call of two new objects $O_{4}$ and $O_{5}$ is received at time $T_{1}^{-}$by base station, it tests the admissibility condition of the code number requirement for the call. At time $T_{1}^{-}$, the code number assigned to $O_{3}$ is released since $O_{3}$ is ended at $T_{1}^{-}$, and the available code number at that time becomes $M_{a}\left(T_{1}^{-}\right)=M_{a}\left(T_{0}\right)+n_{30}=48$. Since this new call failed in the test, the base station proceeds for dropping uniformly some SIU's of $O_{4}$ and $O_{5}$ by performing either NLP1 of (48) or NLP2 of (50). By (51), it leads to these two new assigned code numbers $n_{41}(=20)$ and $n_{51}(=28)$. However, the code numbers for old objects $O_{1}$ and $O_{2}$ during this new time interval $\left[T_{1}, T_{2}\right)$ are given by $n_{11}=n_{10}=26$ and $n_{21}=n_{20}=22$. Finally, the appropriate code numbers for the remaining objects $O_{6}$ and $O_{7}$ during time intervals $\left[T_{2}, T_{3}\right.$ ), $\left[T_{3}, T_{4}\right)$, and $\left[T_{4}, T_{5}\right)$ can be obtained by the proposed assignment procedure and are shown in Fig. 6(b).

\section{CONCLUSION}

In this paper, we have proposed a multicode SS-CDMA system to provide multiple access and retrieval of multimedia information via mobile radio channels interconnecting with the wireline B-ISDN networks. Since the maximum available number of spreading codes in the multicode CDMA system is limited, the objective is to appropriately manage the limited codes of the network for maximum utilization while guaranteeing synchronized presentation of multimedia information within the quality constraints specified by a given BER. Sometimes, since the total code number requirement may exceed the maximum available code number in a time interval, some SIU's of multimedia information should be dropped within a reliability requirement in order to make the transmission rate of multimedia data stream over the mobile radio channel be matched with the playout rate at the mobile terminal. Toward this end, we present a dynamic code assignment scheme to determine both the total droppage of multimedia objects uniformly over all their SIU's and an appropriate code number assigned to each of them. 


\section{REFERENCES}

[1] M. Schwartz, "Network management and control issues in multimedia wireless networks," IEEE Personal Commun. Mag., vol. 2, pp. 8-16, June 1995.

[2] D. Raychaudhuri and N. Wilson, "Multimedia transport in next-generation personal communication networks," in Proc. Int. Conf. Communications, May 1993, pp. 858-862.

[3] T. D. C. Little and A. Ghafoor, "Multimedia synchronization protocols for broadband integrated services," IEEE J. Select. Areas Commun., vol. 9, pp. 1368-1382, Dec. 1991.

[4] M. Woo, N. U. Qazi, and A. Ghafoor, "A synchronization framework for communication of pre-orchestrated multimedia information," IEEE Network Mag., vol. 8, pp. 52-61, Jan./Feb. 1994.

[5] M. Woo, N. Prabhu, and A. Ghafoor, "Dynamic resource allocation for multimedia services in mobile communication environments," IEEE J. Select. Areas Commun., vol. 13, pp. 913-922, June 1995.

[6] A. J. Viterbi, CDMA: Principles of Spread Spectrum Communications. Reading, MA: Addison-Wesley, 1995.

[7] M. H. Fong, Q. Wang, and V. K. Bhargava, "Concatenated orthogonal/PN codes for DS-CDMA systems in a multi-user and multipath fading environment," in Proc. IEEE GLOBECOM'94, Nov. 1994, pp. $1642-1646$

[8] _ "Concatenated orthogonal/PN spreading code scheme for cellular DS-CDMA system with integrated traffic," in Proc. 1995 Int. Conf. Communications, Seattle, WA, June 1995, pp. 905-909.

[9] C. L. I. et al., "Performance of multi-code CDMA wireless personal communication networks," in Proc. 1995 IEEE 45th Vehicular Technology Conf., Chicago, IL, pp. 907-911.

[10] J. G. Proakis, Digital Communications. New York: McGraw-Hill, 1983.

[11] M. B. Pursley, "Spread spectrum multiple access communications," in Multi-User Communication Systems, New York: Springer-Verlag, 1981, pp. 139-189.

[12] D. G. Luenberger, Linear and Nonlinear Programming. Reading, MA: Addison-Wesley, 1984.

[13] M. Kavehrad and B. Ramamurthi, "Direct-sequence spread spectrum with DPSK modulation and diversity for indoor wireless communications," IEEE Trans. Commun., vol. COM-35, pp. 224-236, Feb. 1987.

[14] M. Khansari, A. Jalali, E. Dubois, and P. Mermelstein, "Robust low bit-rate video transmission over wireless access systems," in Proc. IEEE ICC, May 1994, pp. 571-575.
[15] R. Wyrwas, M. J. Miller, R. Anjaria, and W. Zhang, "Multiple access options for multi-media wireless systems," in Proc. Third Workshop Third Generation Wireless Information Networks, Apr. 1992, pp. 289-294.

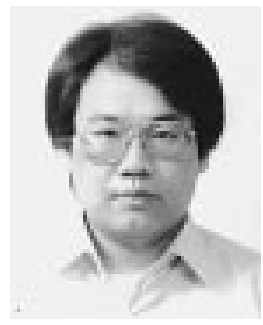

Po-Rong Chang (M'87) received the B.S. degree in electrical engineering from the National Tsing-Hua University, Hsinchu, Taiwan, R.O.C., in 1980, the M.S. degree in communication engineering from National Chiao-Tung University, Hsinchu, Taiwan, R.O.C., in 1982, and the Ph.D. degree in electrical engineering from Purdue University, West Lafayette, IN, 1988, respectively

From 1982 to 1984, he was a Lecturer in the Chinese Air Force Telecommunication and Electronics School for his two-year military service. From 1984 to 1985 , he was an Instructor of electrical engineering at National Taiwan Institute of Technology, Taipei, Taiwan, R.O.C. From 1989 to 1990, he was a Project Leader in charge of SPARC chip design team at ERSO of Industrial Technology and Research Institute, Chu-Tung, Taiwan, R.O.C. Currently, he is a Professor of communication engineering at National Chiao-Tung University. His current interests include wideband CDMA systems, wireless multimedia communications, fuzzy neural networks, and low-power design for wireless communications.

Dr. Chang was the recipient of the Best Paper Award in quality control for semiconductor manufacturing in 1990, Taiwan, R.O.C

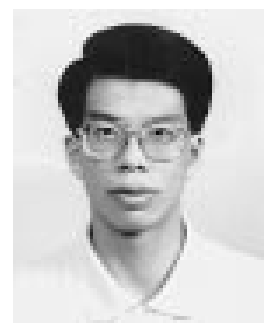

Chin-Feng Lin was born in Taiwan, in 1965. He received the B.S. degree in electrical engineering from Chung-Yung University in 1996 and the M.S. degree in electrical engineering from Chung-Hua University in 1998. Currently, he is working toward the Ph.D degree in communication engineering at the National Chiao-Tung University, Hsinchu, Taiwan, R.O.C.

His research interests include CDMA systems and wireless multimedia communications. 